\title{
Méthodes de Coupe et de Préparation du Terrain Pour Favoriser la Régénération Naturelle de Quelques Tremblaies de L'Est du Québec
}

\author{
René Doucet \\ Service de la Recherche \\ Ministère des Terres et Forêts du Québec
}

\begin{abstract}
Résumé
On a évalué l'état de la régénération du peuplier faux-tremble à la suite de la coupe à blanc conventionnelle, de la coupe totale, et de traitements destinés à stimuler la production de drageons. La coupe totale a donné des résultats très satisfaisants lorsque le peuplement original comprenait au moins $5 \mathrm{~m}^{2} / \mathrm{ha}$ de surface terrière en peupliers biens distribués sur la superficie à régénérer. Cependant, lorsque la distribution de ceux-ci n'était pas adéquate, la régénération comportait d'assez grandes trouées même avec un coefficient de distribution des drageons aussi élevé que 65 p. 100.
\end{abstract}

\begin{abstract}
Aspen reproduction was measured following conventional clearcuts, complete clearcuts, and/or the application of treatments designed to increase the production of root suckers. Regeneration was more than adequate following complete clearcuts, provided that the original stands contained at least $5 \mathrm{~m}^{2} / \mathrm{ha}$ of basal area in aspen uniformly distributed over the area to be regenerated. When aspen distribution was not adequate, even a stocking of 65 p. 100 could mean that fairly large gaps existed in the regeneration.
\end{abstract}

L'utilisation du peuplier a jusqu'à tout récemment été peu importante au Québec; mais à cause de la demande toujours croissante pour les produits forestiers, cette situation change graduellement. Plusieurs utilisateurs ont manifesté de l'intérêt pour cette essence abondante située à proximité des usines; le jour n'est peut-être pas loin où cette ressource passera, dans certaines régions, d'un état de surabondance à celui d'utilisation intensive (Gauthier, Poulin et Thériault, 1975).

Bien que le peuplier faux-tremble (Populus tremuloides Michx), si l'on juge par l'abondance des peuplements actuels, se régénère généralement bien à la suite de perturbations, un aménagement plus intensif amènera inévitablement un besoin d'améliorer la qualité de cette régénération, spécialement dans le cas des peuplements dégradés qui devront faire l'objet de coupes de conversion pour produire sur une période de révolution acceptable, un volume ligneux satisfaisant. Une étude a donc été entreprise afin d'évaluer la régénération naturelle des tremblaies après coupe et de comparer diverses techniques de stimulation de la régénération. Cette étude s'insère dans un programme plus vaste de recherche et de développement sur le peuplier (Vallée, 1972).

\section{La régénération du peuplier faux-tremble}

II semble que le tremble se régénère la plupart du temps par drageonnement (Brinkman et Roe, 1975; Steneker, 1976), à partir de racines situées à moins de $15 \mathrm{~cm}$ de la surface du sol (Maini, 1968). Toute perturbation qui supprime la dominance apicale des tiges, comme la coupe à blanc, le scarifiage, le feu, a pour effet de stimuler la production de drageons (Steneker, 1974); une fois cette dominance supprimée, l'élévation de la température du sol favorisera la production d'un plus grand nombre de drageons.
Stoeckeler et Macon (1956) ont observé que des aires de coupe légèrement ombragées portaient près de 7000 drageons par hectare alors que les aires fortement ombragées en contenaient moins de 1000; ils en on conclu que la densité du peuplement résiduel ne devait pas dépasser $4 \mathrm{~m}^{2} / \mathrm{ha}$ de surface terrière pour assurer une régénération adéquate en peupliers.

La stimulation du drageonnement par le feu a été attribuée à l'absorption de chaleur solaire par la surface noircie du sol; son effet dure généralement une seule année. L'intensité du feu a aussi son importance (Horton et Hopkins, 1966; Maini et Horton, 1966), un feu modéré ayant un effet plus marqué sur la production de drageons qu'un feu léger.

Le scarifiage, en causant des blessures aux racines de l'arbre-mère, stimule la production de drageons; ainsi, en une occasion, cette méthode a favorisé la production de trois à dix fois plus de drageons qu'un terrain non traité, après une année de croissance (Zillgitt, 1951). L'abondance de la régénération est aussi affectée défavorablement par la compétition des broussailles et le jeune âge des parents, et favorisée par la productivité de la station (Stoeckeler et Macon, 1956). Quant à l'effet de la saison, on a rapporté une plus grande abondance de la régénération parfois pour les coupes d'été (Bella et DeFranceschi, 1972) et parfois pour les coupes d'hiver (Zehngraff, 1949; Zasada, 1950), mais l'importance pratique de ces différences semble plutôt négligeable. La présence de drageons uniformément distribués sur toute la superficie au taux de 10,000/ha à l'âge de deux ans (Perala, 1977) ou de 6,000/ha à trois ans (Steneker, 1976) a été jugée nécessaire à l'obtention d'un peuplement complet, car les pertes par mortalité sont très grandes au cours des premières années.

\section{Méthodes}

\section{Traitements en vue de stimuler le drageonnement}

Cette étude a été réalisée sur une superficie d'environ 14 ha, dans le canton Ouimet, comté de Rimouski, Québec. Ce territoire fait partie de la section Témiscouata-Restigouche (L. 6) de la région forestiere des Grands-Lacs et du SaintLaurent (Rowe, 1972). Le sol est un till mince à partir duquel se développe un podzol; des affleurements rocheux sont visibles par endroits. La végétation provient d'un feu de 1930 et se classe soit dans la série de la sapiniere à bouleau jaune, soit dans celle de la sapiniere à thuya dans les dépressions humides. Les peupliers faux-trembles et baumiers (Populus balsamifera L.) présents avant la coupe étaient les vestiges du stade pionnier de la tremblaie, les autres essences les plus abondantes étant le bouleau à papier (Betula papyrifera Marsh.), l'épinette blanche (Picea glauca (Moench.) Voss) et le sapin baumier (Abies balsamea (L) Mill.).

D'après la hauteur des peupliers dominants, $20 \mathrm{~m}$ à 40 ans, la station appartient à la classe de fertilité I. Cependant, le volume marchand (tiges de $9 \mathrm{~cm}$ et pluș au d.h.p.) de $109,5 \mathrm{~m}^{3} /$ ha représentait à peine 50 p. 100 du volume normal calculé pour les pleupleraies du Bas-Saint-Laurent (Le Goff et al., 1976). Ce volume se composait à 37 p. 100 de 
peupliers, 36 p. 100 d'autres feuillus et 27 p. 100 de résineux. Le nombre total de tiges de $1,5 \mathrm{~cm}$ et plus de d.h.p. s'élevait à $2267 / \mathrm{ha}$, la surface terriere à $24,2 \mathrm{~m}^{2} / \mathrm{ha}$ et le diamètre moyen à $11,7 \mathrm{~cm}$; les mesures correspondantes pour le peuplier étaient: 236 tiges/ha, $6,0 \mathrm{~m}^{2} /$ ha et $18,0 \mathrm{~cm}$. De plus les morts-bois, principalement l'érable à épis (Acer spicatum Lam.) et le cerisier de Pennsylvanie (Prunus pensylvanica L. f.), représentaient près de 3000 tiges/ha. On était donc en présence d'un peuplement de qualité médiocre.

Le territoire choisi a été divisé en sept secteurs de 1,0 ha de superficie, séparés les uns des autres par une bande de protection de $40 \mathrm{~m}$ de largeur. Chacun a reçu quatre lignes d'inventaire, distribuées au hasard, qui ont servi à mesurer les caractéristiques dendrométriques du peuplement original. On a ensuite établi au hasard, sur chaque ligne, quatre grappes de dix quadrats de $4 \mathrm{~m}^{2}$ de superficie, pour un total de 160 quadrats par secteur (fig. 1). Dans le premier quadrat de chaque grappe, on a dénombré la régénération par essence et classe de hauteur, tandis que pour les neuf autres, on s'est contenté d'en noter la présence ou l'absence par essence, pour les classes de hauteur suivantes: 15 a $45 \mathrm{~cm}, 46$ a $110 \mathrm{~cm}$ et $111 \mathrm{~cm}$ et plus. Avec ces données, on a calculé le coefficient de distribution de la régénération, c'est-à-dire le pourcentage des quadrats régénérés. On a effectué cet inventaire immédiatement avant la coupe et chaque année pour les cinq années suivantes, sauf la troisieme.

Deux secteurs ont été conservés intacts pour suivre l'évolution du peuplement. On a exploité les cinq autres à l'automne 1971, toutes les tiges de $1,5 \mathrm{~cm}$ et plus de d.h.p. étant abattues. Dans l'un de ceux-ci, on a enlevé les déchets de coupe, un autre a été scarifié au printemps 1972 au moyen d'un scarificateur à barils munis d'ailerons, et les trois derniers ont été scarifiés après qu'on ait éliminé les déchets par brûlage en tas. Ce dernier traitement était représenté par trois secteurs afin que l'on puisse, plus tard, effectuer dans un secteur une éclaircie pré-commerciale et dans un autre de la fertilisation.

\section{Inventaire d'aires de coupe}

Pour compléter l'étude, on a fait l'inventaire de 6 aires de coupe de moins de 10 ans, dans les tremblaies à résineux de la région du Bas-Saint-Laurent; trois avaient été soumises à une coupe à blanc conventionnelle, c'est-à-dire que les gros peupliers et les résineux avaient été enlevés, et les trois autres avaient fait l'objet d'une coupe totale des tiges de $1,5 \mathrm{~cm}$ et plus de d.h.p.

Dans chaque aire, on a établi au moins 40 grappes de 10 quadrats de la manière décrite précédemment. On a noté le nombre de quadrats recouverts de déchets de coupe, l'épaisseur de l'humus, la texture du sol, l'abondance des broussailles, la nature du peuplement original, ainsi que la hauteur et l'âge de cinq drageons dominants de peuplier. Enfin, on a inventorié le peuplement résiduel dans des placettes carrées de 0,04 ha de superficie.

\section{Résultats et Discussion}

En combinant les données obtenues par les inventaires d'aires de coupe à celles des traitements destinés à stimuler le drageonnement, on peut examiner les résultats des cinq traitements suivants: coupe à blanc conventionnelle, coupe totale, élimination des déchets plus scarifiage.

La régénération en peupliers dans les aires de coupe totale est amplement suffisante pour former un peuplement complet (tableau 1); elle est plus variable dans les aires de coupe à blanc conventionnelle, même si dans tous les cas le nombre de tiges de peupliers du peuplement original était suffisant pour produire une quantité de drageons adéquate. Ceci pourrait s'expliquer, soit par la présence des tiges résiduelles qui provoqueraient la mortalité des drageons par suppression, soit par le fait qu'un nombre restreint de tiges de peupliers ont été coupées, diminuant ainsi la production de drageons. Cette situation pourrait toutefois permettre à la régénération résineuse, parfois abondante, d'occuper une place plus importante dans le futur peuplement.

Les autres variables mesurées, soit l'importance des déchets de coupe, l'épaisseur de l'humus, la texture du sol et l'abondance des morts-bois, ne permettent pas d'expliquer les variations rencontrées dans la régénération, probablement parce que les conditions étaient relativement uniformes dans toutes les aires de coupe.

Contrairement aux aires de coupe analysées ci-dessus, les techniques pour stimuler la production de drageons ont été appliquées dans un peuplement qui contenait une faible proportion de peupliers et pratiquement pas de régénération résineuse. Un an après les traitements, le nombre de drageons de peupliers variait d'un peu plus de 14000 à plus de 140000 par hectare, avec un coefficient de distribution de 50 à 98 p. 100. Quatre ans plus tard, ce nombre était environ dix fois moindre, mais le coefficient de distribution avait
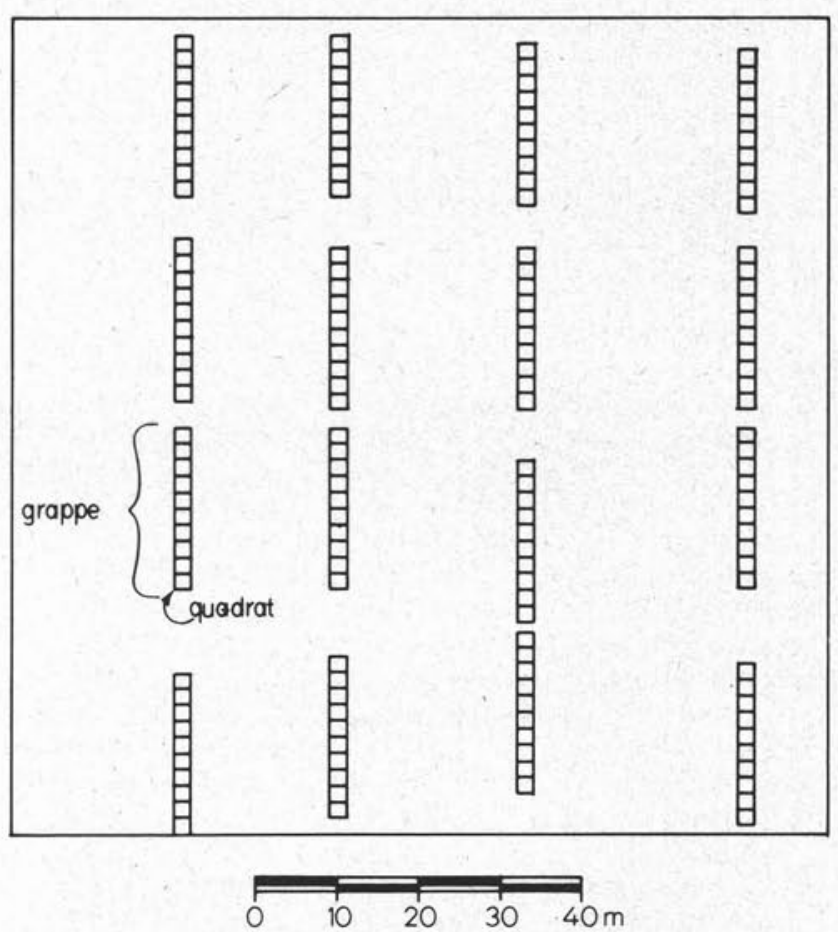

Fig. 1. Agencement des quadrats dans un secteur.

Tableau 1. Régénération après coupe dans quelques aires de coupe

\begin{tabular}{|c|c|c|c|c|c|}
\hline \multirow{3}{*}{$\begin{array}{c}\text { Age de } \\
\text { la coupe } \\
\left.\text { ( } \mathrm{m}^{2} / \mathrm{ha}\right)\end{array}$} & \multirow{3}{*}{\multicolumn{2}{|c|}{$\begin{array}{l}\text { Surface terrière } \\
\text { résiduelle } \\
\text { coefficient } \\
\text { de distribu- } \\
\text { tion }(\%)\end{array}$}} & \multicolumn{3}{|c|}{ Régénération } \\
\hline & & & \multicolumn{2}{|c|}{ Peuplier } & Résineux \\
\hline & & & tiges/ha & $\begin{array}{l}\text { coefficient } \\
\text { de distribu- } \\
\text { tion }\end{array}$ & tiges/ha \\
\hline 2 & 0 & 84,4 & 30815 & 39,7 & 7265 \\
\hline 3 & 0 & 83,1 & 30400 & 6,3 & 408 \\
\hline 5 & 0 & 82,7 & 9435 & 27,3 & 4205 \\
\hline 2 & 8,6 & 70,8 & 11785 & 63,8 & 8654 \\
\hline 4 & 4,8 & 36,2 & 3420 & 74,6 & 12885 \\
\hline 7 & 6,4 & 76,9 & 8880 & 15,0 & 2656 \\
\hline
\end{tabular}


diminué assez peu, surtout dans les secteurs les mieux régénérés (tableau 2). La diminution du nombre de tiges a été très forte entre la première et la deuxième année, quel que soit le nombre initial, puis plus graduelle par la suite (fig. 2).

Plusieurs autres essences feuillues, notamment le cerisier de Pennsylvanie, étaient présentes immédiatement après la coupe. Elles aussi ont subi une baisse importante, de sorte que le nombre total de tiges, assez semblable d'un secteur à l'autre, est passé d'environ 175000 à 26000 par hectare au cours des cinq premières années après la coupe. II est donc important, comme l'indique Steneker (1976) que les drageons soient abondants afin d'occuper le plus possible le terrain; ils devraient alors être en mesure de surmonter la concurrence des morts-bois, car après cinq ans on les retrouve essentiellement dans la classe de hauteur supérieure ( $111 \mathrm{~cm}$ et plus) alors qu'une proportion non négligeable de leurs concurrents se recrute dans les classes de hauteur inférieure.

Le coefficient de distribution ne donne pas toujours une image exacte de l'état de la régénération. Ainsi le secteur ayant un coefficient global de 65 p. 100 après cinq ans (tableau 2), est formé en réalité de superficies bien régénérées dont le coefficient de distribution est de 88 p. 100, et de

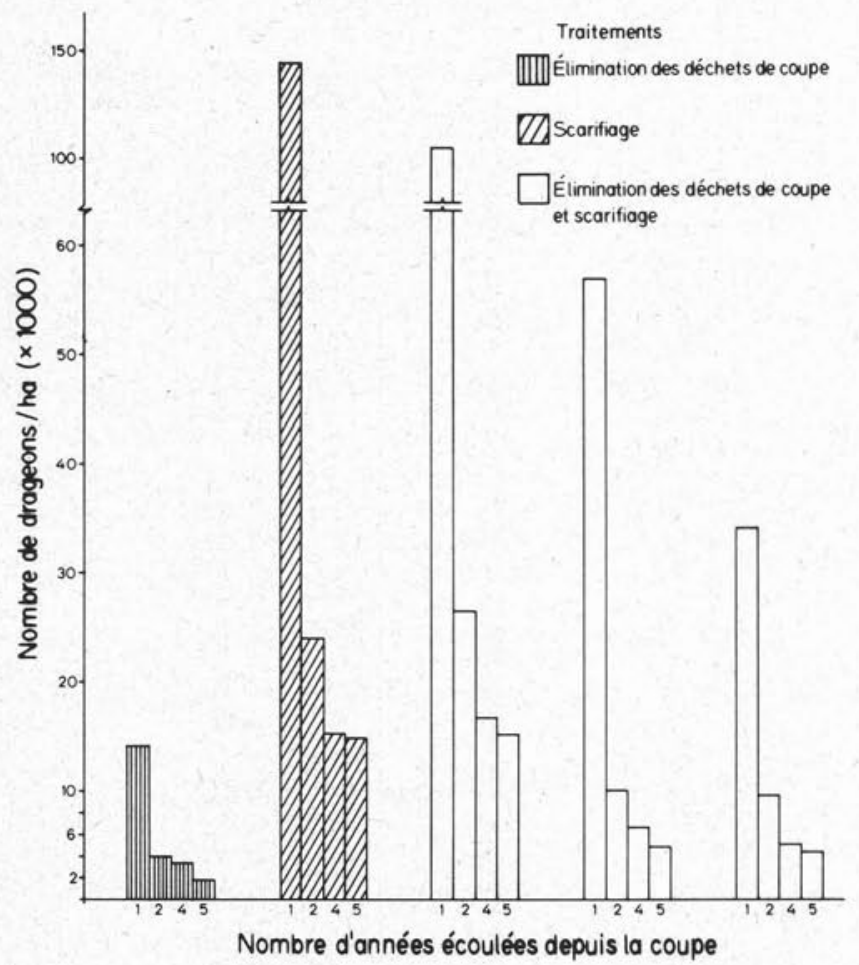

Fig. 2. Diminution du nombre de drageons en fonction des années écoulées depuis la coupe. trouées mal régénérées dont le coefficient de distribution tombe à 14 p. 100, sans intermédiaire entre ces deux situations. Donc, même avec un coefficient de distribution global qui semble adéquat, car un coefficient de 55 p. 100 après cinq ans est souvent considéré comme satisfaisant (Stoeckeler et Macon, 1956; Heeny et al., 1975), le pourcentage de superficie mal régénérée est de $30 \mathrm{p}$. 100 dans ce cas particulier. La situation est même plus grave dans les deux autres secteurs moins bien régénérés.

II ne semble pas que les divers traitements aient stimulé la production de drageons à un niveau plus élevé que celui de la coupe totale, et à tout le moins ils n'ont pas empêché que des trouées importantes se développent dans certains secteurs. La présence de drageons semble plutôt liée à celle des tiges de peupliers dans le peuplement original: les secteurs contenant un nombre suffisant de tiges ont produit des drageons en abondance quel que soit le traitement appliqué. Ce niveau requis peut être défini au moyen des figures 3 et 4 qui présentent les relations entre le coefficient de distribution d'une part, et d'autre part la surface terrière et le nombre de tiges du peuplier avant coupe. On y voit qu'un coefficient de distribution de 75 p. 100 correspond à une surface terrière avant coupe de $5,3 \mathrm{~m}^{2} / \mathrm{ha}$ et à 165

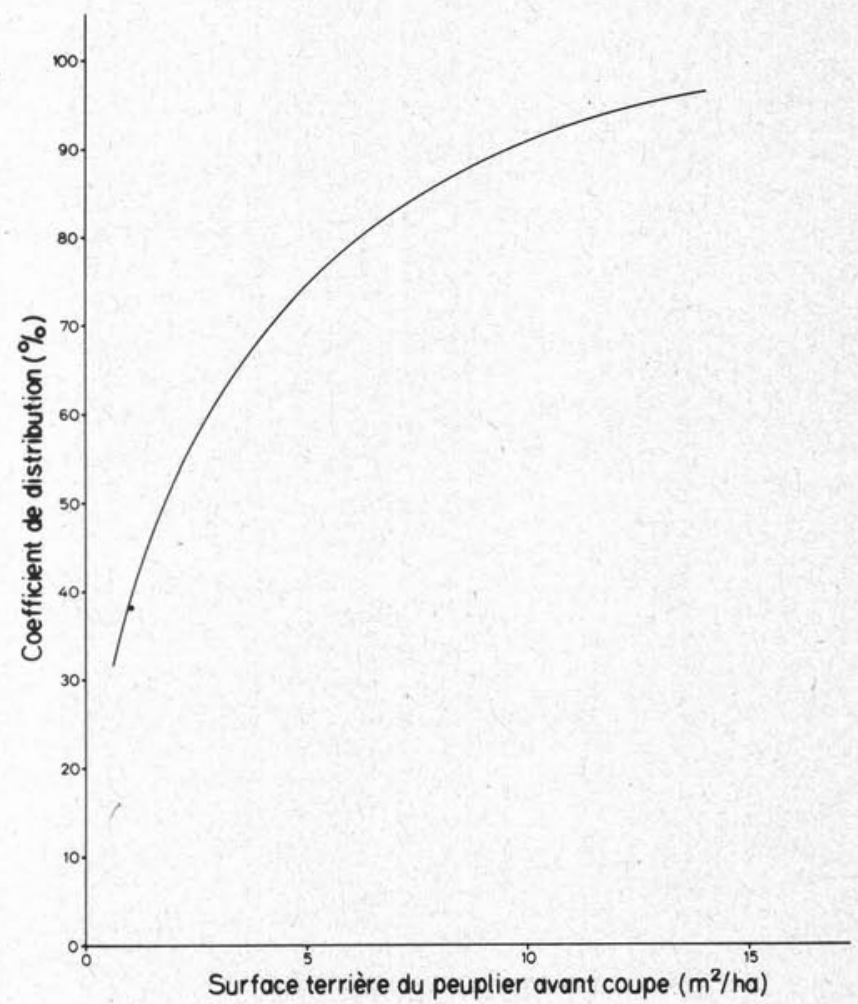

Fig. 3. Variation du coefficient de distribution, cinq ans après la coupe, en fonction de la surface terrière en peuplier du peuplement original.

Tableau 2. Regénération dans des secteurs soumis à divers traitements dans le but de stimuler le drageonnement du peuplier

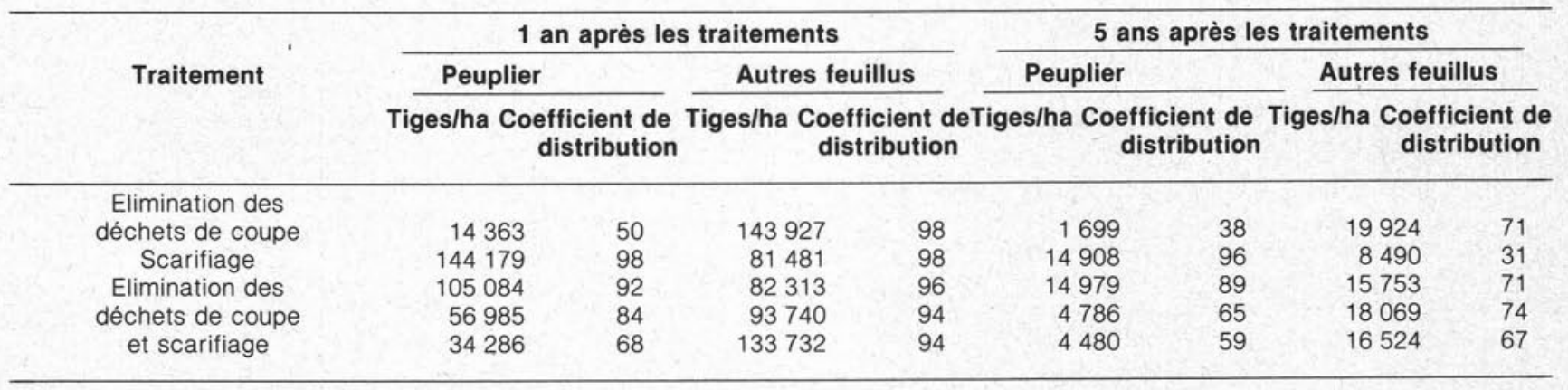


tiges/ha, ce qui donne un espacement moyen de 7,8 $\mathrm{m}$ pour des tiges d'un diamètre moyen de $17,9 \mathrm{~cm}$. II est intéressant de noter que, selon Perala, un peuplement doit contenir au moins 126 tiges/ha de peupliers, pour une surface terrière de $5 \mathrm{~m}^{2} /$ ha et un espacement d'environ $9 \mathrm{~m}$, pour obtenir une régénération adéquate. Cet espacement moyen peut varier selon le degré d'uniformité de la distribution des tiges, mais il constitue un guide valable, d'autant plus que Brinkman et Roe (1975) soutiennent que la majorité des drageons naissent à moins de $8 \mathrm{~m}$ de l'arbre-mère.

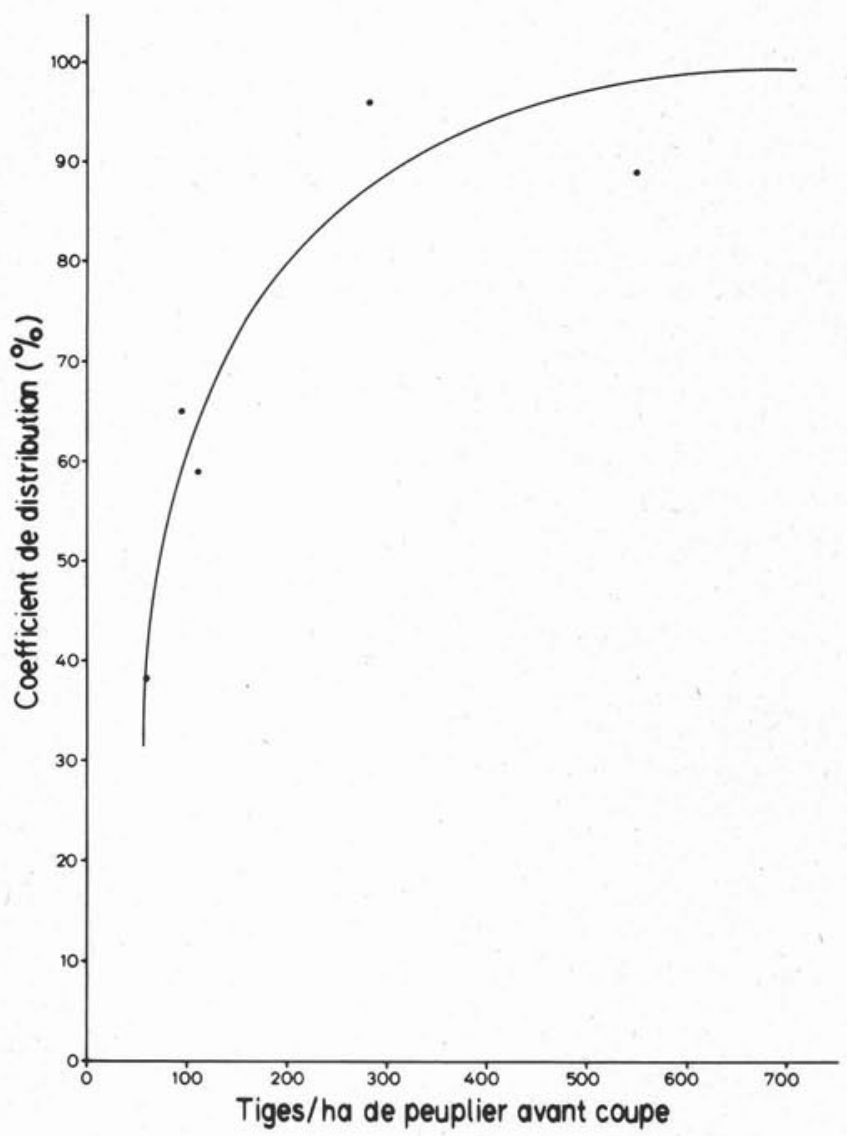

Fig. 4. Variation du coefficient de distribution, cinq ans après la coupe, en fonction du nombre de tiges de peuplier du peuplement original.

\section{Conclusion}

Pour assurer la régénération du peuplier dans les peuplements où cette essence occupe une place importante, il suffit généralement que la coupe soit la plus complète possible. Par contre, la régénération des peuplements dégradés contenant une quantité plutôt faible de peupliers, présente un problème particulier. Une bonne régénération peut être obtenue avec aussi peu que $5 \mathrm{~m}^{2} /$ ha de surface terrière en peupliers avant la coupe, à la condition que l'espacement entre les tiges ne dépasse pas 8 à $10 \mathrm{~m}$. De plus, un coefficient de distribution de 75 p. 100 après cinq ans devrait être adopté comme norme pour juger du succès de la régénération.
II ne semble pas que des travaux de stimulation de la régénération puissent être recommandés, car dans ce type de peuplement, l'absence de régénération en peupliers est le plus souvent liée à la trop faible représentation des tiges du peuplier dans le peuplement avant coupe, de sorte qu'il en résultera d'importantes trouées envahies par les mortsbois, si les tiges de peupliers du peuplement original sont trop espacées.

\section{Remerciements}

Nous désirons souligner la contribution de messieurs Carol LaBarre, ing.f., qui a préparé ce projet et mis en place le dispositif expérimental, et Gilles Gignac, tech. for., qui a procédé aux mesurages annuels.

\section{Bibliographie}

Bella, I. E., et J. P. De Franceschi, 1972. The effect of logging practices on the development of new aspen stands, Hudson Bay, Saskatchewan. Serv. Can. For., Rapp. Inf. NOR-X-33. 20 p.

Brinkman, K. A., et E. I. Roe, 1975. Quaking aspen: silvics and management in the Lake States. U.S. For. Serv., Agric. Handbook No $486.52 \mathrm{p}$.

Gauthier, Poulin, Thériault et Assoc., 1975. Le marché du peuplier au Québec, 1985-1995. Min. Terres et Forêts du Québec, Serv. Restauration. 76 p.

Heeney, C. J., J. A. Kemperman et G. Brown, 1975. A silvicultural guide to the aspen working group in Ontario. Ont. Min. Nat. Res., Div. Forests. $47 \mathrm{p}$.

Horton, K. W., et E. J. Hopkins, 1966. Influence of fire on aspen suckering. Serv. can. For., Publ. No 1095. 19 p.

Le Goff, N., M. Ménard et Y. Richard, 1976. Recherche et développement sur le peuplier dans la région de l'Est du Québec. VIII - Tables de rendement pour les peupleraies naturelles dans les comtés de Montmagny à Rimouski. Min. Ter. et For., Serv. de la rech., Mémoire No 32.80 p.

Maini, J. S., 1968. Silvics and ecology of Populus in Canada. In: growth and utilization of poplars in Canada. Min. For. Devel. Rural, Div. For., Publ. No 1205: 20-69.

Maini, J. S., et K. W. Horton, 1966. Reproductive response of Populus and associated Pteridium to cutting, burning and scarification. Serv. Can. For., Publ. No 1155.20 p.

Perala, D. A., 1977. Aspen in the North-Central States. U.S. For. Serv., Gen. Tech. Rep. NC-36. 30p.

Rowe, J. S., 1972. Les régions forestières du Canada. Serv. Can. For., Publ. No 1300F. $171 \mathrm{p}$.

Steneker, G. A., 1974. Factors affecting the suckering of trembling aspen. For. Chron. 50: 32-34.

1976. Guides to the silvicultural management of trembling aspen in the prairie provinces. Serv. Can. For., Rapp. Inf. NOR-X-164. $6 \mathrm{p}$.

Stoeckeler, J. H., et J. W. Macon, 1956. Regeneration of aspen cutover areas in Northern Wisconsin. J. For. 54: 13-16.

Vallée, G., 1972. Recherche et développement sur le peuplier dans la région de l'est du Québec. I - Présentation des projets. Min. Ter. et For., Serv. de la rech., Mémoire No 8. 115 p.

Zasada, Z. A., 1950. Aspen management problems of the Lake States. U.S. For. Serv., Lake States For. Exper. Sta., Misc. Rep. No 10.23 p.

Zehngraff, W. M., 1949. Aspen as a forest crop. J. For. 47: 555-565.

Zillgitt, W. M., 1951. Disking to increase stocking in aspen stands. U.S. For. Serv., Lake States For. Exper. Sta., Tech., Note No 357. 